\title{
Organic-Inorganic Hybrid Thermoelectric Material Synthesis and Properties
}

\author{
Jiwon Kim and Jae-Hong Lim ${ }^{\dagger}$ \\ Electrochemistry Research Group, Materials Processing Division, Korea Institute of Materials Science, Changwon 51508, Korea \\ (Received July 14, 2017; Revised July 19, 2017; Accepted July 19, 2017)
}

\begin{abstract}
Organic-inorganic hybrid thermoelectric materials have obtained increasing attention because it opens the possibility of enhancing thermoelectric performance by utilizing the low thermal conductivity of organic thermoelectric materials and the high Seebeck coefficient of inorganic thermoelectric materials. Moreover, the organic-inorganic hybrid thermoelectric materials possess numerous advantages, including functional aspects such as flexibility or transparency, low cost raw materials, and simplified fabrication processes, thus, allowing for a wide range of potential applications. In this study, the types and synthesis methods of organic-inorganic thermoelectric hybrid materials were discussed along with the methods used to enhance their thermoelectric properties. As a key factor to maximize the thermoelectric performances of hybrid thermoelectric materials, the nanoengineering to control the nanostructure of the inorganic materials as well as the modification of the organic material structure and doping level are considered, respectively. Meanwhile, the interface between the inorganic and organic phase is also important to develop the hybrid thermoelectric module with excellent reliability and high thermoelectric efficiency in addition to its performance in various electronic devices.
\end{abstract}

Key words: Thermoelectric, Organic-inorganic hybrid thermoelectric materials, Nanoengineering, Thermoelectric properties

\section{Introduction}

$\mathrm{T}$ he depletion of fossil fuels and growing environmental pollution have sparked global interest in renewable energy development. Among the various approaches, thermoelectric energy conversion devices in particular have received consistent interest in the renewable energy field, because they can efficiently maximize the utilization of waste heat from fossil fuel use. To realize high efficiency, studies continue to be carried out to investigate and develop more advanced thermoelectric materials and devices. Also, considering the increased mobility and flexibility requirements of future electronic devices, the next generation thermoelectric material needs to be both highly efficient and flexible. To be applicable to diverse forms of future electronic devices and equipment, the thermoelectric material should be adaptable to various installations and forms. The performance of thermoelectric materials is defined by the figure of merit $\mathrm{ZT}=\mathrm{S}^{2} \sigma \mathrm{T} / \kappa$. Here, $\mathrm{S}, \sigma, \mathrm{T}$, and $\kappa$ refer to the Seebeck coefficient, electrical conductivity, absolute temperature, and thermal conductivity. The performance of the thermoelectric material is higher when the thermal conductivity is low and the electrical conductivity and Seebeck coefficient are high. Accordingly, to develop a material with

\footnotetext{
Corresponding author: Jae-Hong Lim

E-mail : lim@kims.re.kr

Tel : +82-55-280-3523 Fax : +82-55-280-3570
}

high thermoelectric efficiency, the electrical conductivity and Seebeck coefficient of the material have to be increased while decreasing its thermal conductivity. However, these material properties have trade-off relationships, so some limiting factors need to be resolved to control the properties independently. ${ }^{1)}$ For example, the Seebeck coefficient is a representative material property that has a trade-off relationship with the carrier concentration, where an increase in the carrier concentration leads to a decrease in the Seebeck coefficient (Fig. 1). On the other hand, the electrical conductivity is improved with greater carrier concentration. Consequently, a material with high thermoelectric efficiency needs to achieve maximum performance within the trade-off relationships between the carrier concentration and the electrical conductivity, along with the Seebeck coefficient. Also, in order to effectively decrease only the thermal conductivity without decreasing the electrical conductivity, the movements of electrons and phonons have to be controlled independently, to decrease just the lattice thermal conductivity. Recently, such control has become possible through the application of inorganic nanostructures of various forms..$^{2-5)}$

Compared to conventional inorganic thermoelectric hybrid materials, it has recently been reported that high efficiency thermoelectric materials were produced through the synthesis of organic-inorganic hybrid thermoelectric materials, which also exhibited properties of light weight and flexibility. This result has garnered increasing attention because it 


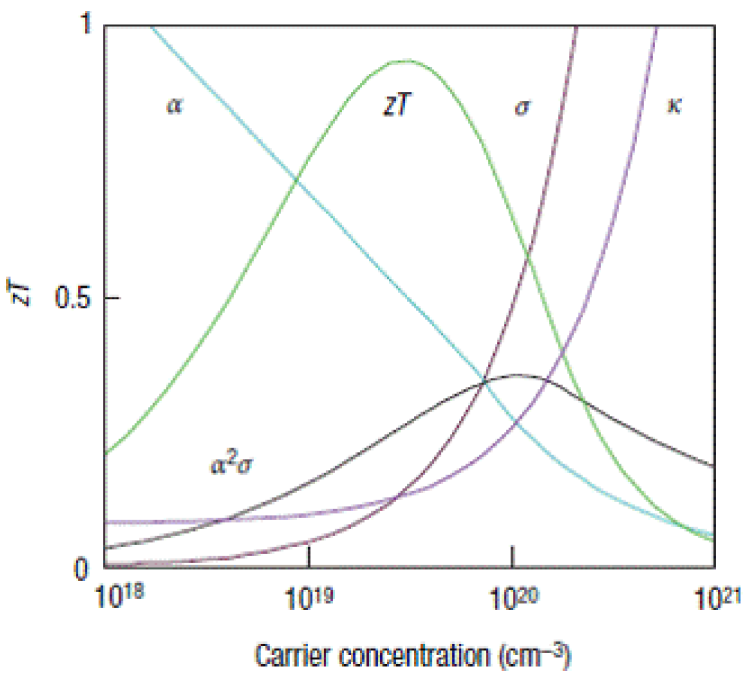

Fig. 1. Thermoelectric property control according to changes in the carrier concentration.

opens the possibility of enhancing thermoelectric performance by utilizing the low thermal conductivity of organic thermoelectric materials and the high Seebeck coefficient of inorganic thermoelectric materials. ${ }^{6,7)}$ Moreover, such organicinorganic hybrid thermoelectric materials possess numerous advantages, including functional aspects such as flexibility or transparency, low cost raw materials, and simplified fabrication processes, thus, allowing for a wide range of potential applications. In this report, recent studies and technological findings are detailed with a focus on the improvement in thermoelectric properties achieved through the synthesis of organic-inorganic hybrid thermoelectric materials with nanostructures.

\section{Synthesis of Low-Dimensional Organic/ Inorganic Hybrid Thermoelectric Material}

In order to effectively enhance the thermoelectric properties of organic-inorganic hybrids, optimization of the thermoelectric properties of each single phase of the organic and inorganic material needs to be carried out in advance. Generally, inorganic thermoelectric materials are fabricated by chemical synthesis, such as the solvothermal method, ball milling, spark erosion, and electrodeposition. Enhancing the thermoelectric performance of inorganic thermoelectric materials is generally approached through the synthesis of nanostructures (Fig. 2) ${ }^{8-12}$ For example, the room temperature thermoelectric properties of nanowires with 1-dimensional structures, and 3-dimensional n-type $\mathrm{Bi}_{2} \mathrm{Te}_{3}$ and $\mathrm{p}$ type $\mathrm{Sb}_{2} \mathrm{Te}_{3}$ nanostructures composed of nanoscale grains, can be improved using the quantum confinement effect, energy filtering effect, and PGEC (phonon glass electron crystal).

Improvements in the properties of conducting polymers, including electrical, optical, and thermoelectric characteristics, have been reported recently and research using such (a)

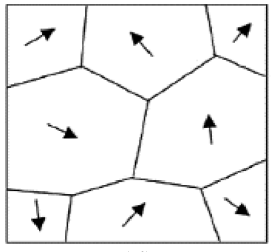

(d)

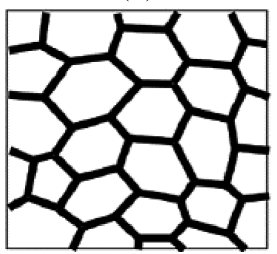

(b)

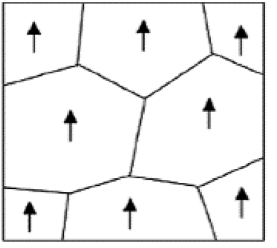

(e)

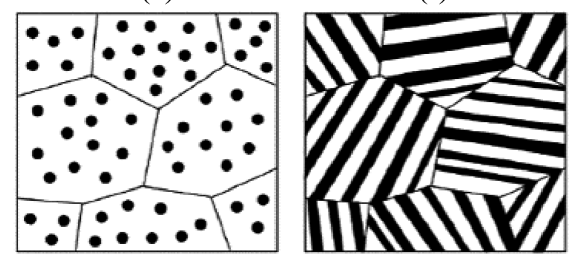

Fig. 2. Improvement of thermoelectric properties through nanostructure synthesis. (a) Polycrystalline microstructure, (b) polycrystalline microstructure composed of grains with directionality, (c) nanostructure composed of nanoscale grains, (d) nanostructure composed of nanocoated grains, (e) nanocomposite containing heterogeneous nano objects, (f) lamellar/ multi-layer nanocomposite

improvements is becoming more significant. ${ }^{13,14)}$ In particular, conducting polymers with high electrical properties such as polypyrrole (PPy), PANI, polythiophene (PTH), poly(3,4-ethylenedioxythiophene) (PEDOT), and polyacetylene $(\mathrm{PA})$ have great potential in thermoelectric materials, having low thermal conductivity and being environment friendly. Accordingly, studies are being actively pursued to improve the polymers' thermoelectric performance by carrying out technology development for more effective synthesis and property control, as well as better understanding of the charge transport mechanism. ${ }^{15)}$ Poly(3,4-ethylenedioxythiophene):polystyrene sulfonate (PEDOT:PSS) is a well-known organic thermoelectric material with high stability at room temperature and atmospheric pressure along with high electrical conductivity and low thermal conductivity. PEDOT: PSS can be synthesized using physical mixing, solution mixing, and in-situ oxidative polymerization, and it has been

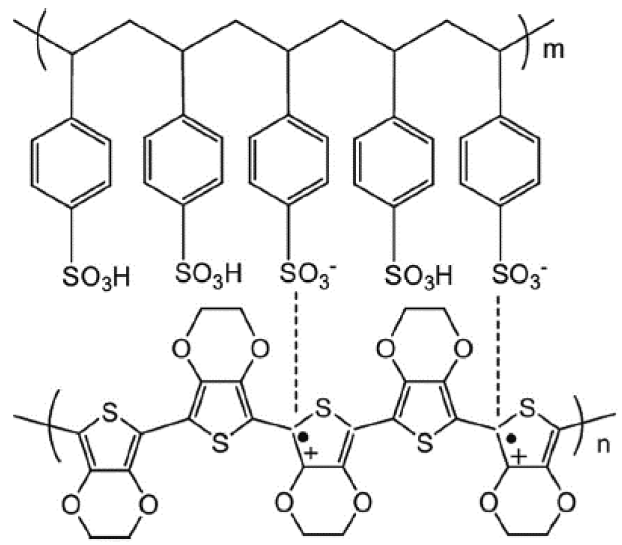

PSS

PEDOT

Fig. 3. PEDOT:PSS chemical structure. 
reported that appropriate control of the synthesis conditions can improve the thermoelectric performance of PEDOT: PSS. $^{16)}$

For example, the electrical conductivity of PEDOT:PSS can be improved by controlling charge mobility based on the PSS interval within the chain. ${ }^{16)}$ This improvement is possible because the PSS acts as a pathway to aid charge transport within the PEDOT chain (Fig. 3). ${ }^{17)}$ However, while the PEDOT:PSS produced using this method has improved electrical conductivity, it does not exhibit a decrease in the Seebeck coefficient, and as a result achieves improved thermoelectric performance.

In addition, the properties of the conducting polymers can be controlled through doping. For example, the doping levels of PPy, PEDOT, and PANI which have been synthesized electrochemically were found to be determined by the electron deficiency of the polymer backbone during the electrochemical oxidation/reduction process. Using this characteristic, Bubnova et al. controlled the oxidation level of PEDOT:p-toluenesulfonate (PEDOT:Tos) through de-doping using tetrakis(dimethylamino)ethylene (TDAE) to show that an improved Seebeck coefficient can be obtained. ${ }^{18)}$ Additionally, Kim et al. used the well-known de-doping material ethylene glycol (EG) to control the PEDOT:PSS oxidation level, to obtain a ZT value of up to 0.42 at room temperature. ${ }^{19)}$ The doping of conducting polymers typically results in improvement in their electrical properties, by modifying their electronic structure, and improvement in their electrical conductivity occurs through the modification of the polymer structure. In general, changing the typical coil structure of a conducting polymer into a well extended linear structure improves its charge mobility, and through this approach, the electrical conductivity of the polymers has been enhanced (Fig. 4). Wang et al. used carbon nanotubes as the platform to synthesize regularly spaced PANI/ CNT composites, and the composite's exhibited improved electrical properties (Fig. 5) because the linear structure of the PANI had increased charge mobility due to the relatively high electron delocalization. ${ }^{20)}$ This was a significant finding, which revealed that improving the crystallinity of a conducting polymer is an effective method of improving its thermoelectric properties. Cho et al. synthesized a selfassembling PEDOT nanowire using vapor phase polymer- ization without using a template. The synthesized PEDOT nanowire had a crystallinity close to a single phase and a high electrical conductivity of $8797 \mathrm{~S} / \mathrm{cm}^{21)}$

Similarly, a thermoelectric hybrid containing organic and inorganic materials with improved electrical conductivity and Seebeck coefficient due to structural modification is expected to exhibit enhanced physical and thermoelectric properties than existing single phase nanostructures. Recently, there were various reports on the high efficiency synthesis of organic-inorganic hybrid thermoelectric materials through the application of various chemical approaches, self-assembly, and nanofabrication. In order to effectively synthesize such hybrid materials, various elements need to be considered. In particular, the characteristic of the interface produced during the combination of the 2 phases is a critical element that can determine the overall electrical and thermoelectric properties of the organic-inorganic hybrid. For example, in addition to the selection of organic and inorganic materials that have suitable differences in work function value, a synthesis method is required that can effectively control the interfacial density within the hybrid, as well as defects existing at the interface. First, the ability of inorganic nanoparticles to be uniformly dispersed in the organic material can have an impact on the reliability and commercialization of the organic-inorganic hybrid. Unfortunately, the dispersant (surfactant, stabilizer) commonly used to enhance nanoparticle dispersion is difficult to completely remove during the synthesis process, and so the dispersant remains as a residue, and can have a negative effect on the hybrid thermoelectric property. To overcome this problem, Lu et al. did not use a dispersant, but instead employed an interfacial polymerization method to synthesize a cable shaped Au/PEDOT nanostructure. ${ }^{22)}$ The PbTePEDOT nanotube, synthesized in a similar manner, produced the maximum power factor without an oxidation reaction at the interface. ${ }^{23)}$ Also, to improve thermoelectric properties by controlling the charge transport mechanism at the interface, the carrier forms of the organic and inorganic materials have to be the same, and the work function values $(\sim 150 \mathrm{meV})$ have to be similar. See at al. synthesized a p-type Te nanorod using an aqueous solution method within an aqueous solution with PEDOT:PSS, so that a PEDOT:PSS coated Te/PEDOT:PSS film type hybrid was (a)

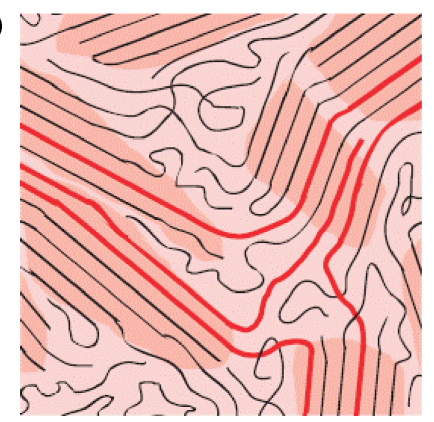

(b)

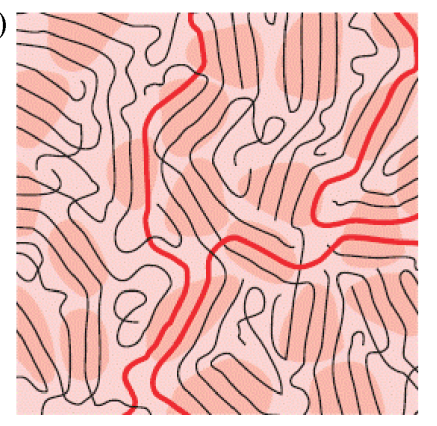

(c)

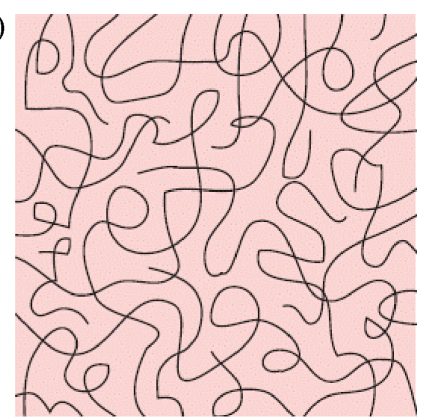

Fig. 4. Conjugate polymer crystallinity diagram. (a) semi-crystalline, (b) amorphous aggregate, (c) amorphous polymer structure. 
(a)

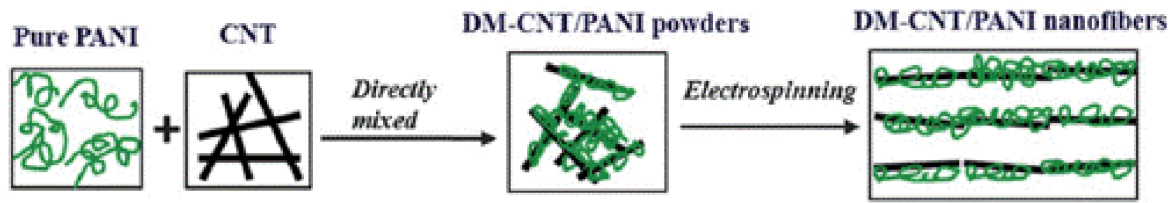

(b)
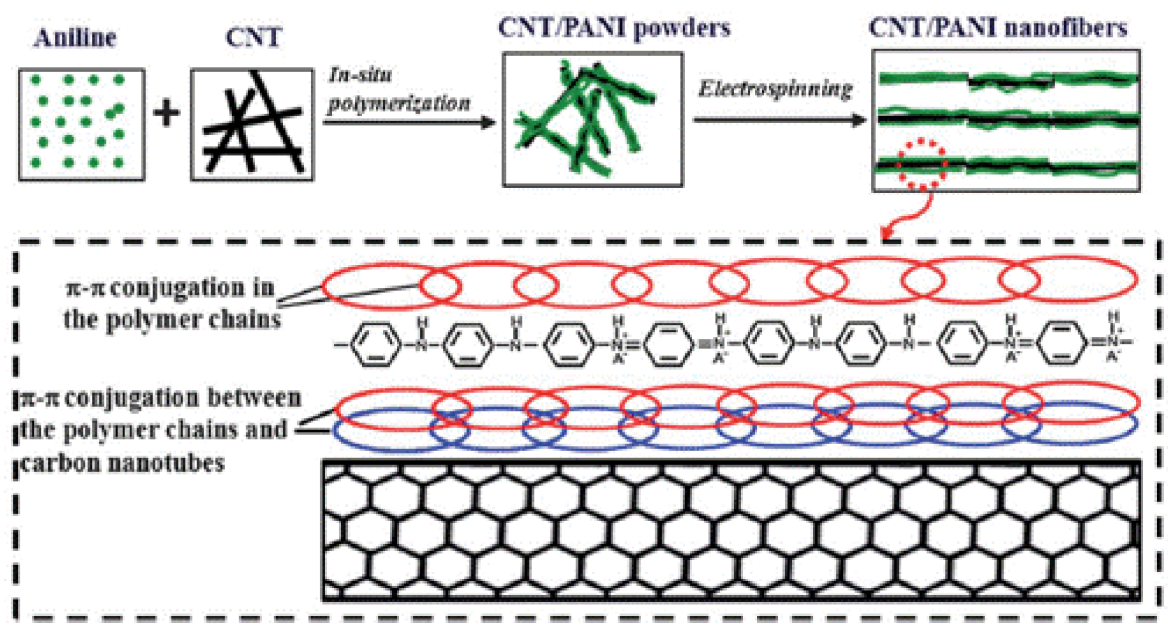

Fig. 5. Synthesis of regularly spaced PANI/CNT hybrid.

synthesized. The Te/PEDOT:PSS showed a ZT value of 0.1 which was approximately 103 times greater than the ZT of the single phase Te nanorod and PEDOT:PSS. This result was due to maintaining the hybrid's high electrical conductivity without a significant decrease in the Seebeck coefficient. This was possible because the high conductivity PEDOT:PSS surrounding the Te nanorod surface was connected with no defects, so that the PEDOT:PSS could effectively perform its role as the charge transport pathway. ${ }^{24)}$

\section{Low-Dimensional Organic/Inorganic Hybrid Thermoelectric Material Property Evaluation and Technology Improvement}

As discussed earlier, the thermoelectric properties of a heterogeneous organic-inorganic hybrid can be improved and avoid the limitations of the trade-off relationships, by engineering of the interfaces that exist within the hybrid. For example, the carrier filtering effect produced by the energy level mismatch between inorganic nanoparticles and an organic material can increase the power factor by improving the independent Seebeck coefficient of the organic-inorganic hybrid. Moreover, the charge transport mechanism can be varied depending on the structural constituent, and alignment of the conducting polymer chain at the interface, which can then contribute to an improvement in thermoelectric efficiency. As an example, the chalcogenide-based $\mathrm{Te}, \mathrm{Bi}_{2} \mathrm{Te}_{3}$, and $\mathrm{PbTe}$, which have been reported to exhibit high thermoelectric performances at room temperature, were synthesized into a nanostructure followed by synthesis with poly(3,4-ethylenedioxythiophene)-poly(styrenesulfonate) (PEDOT:PSS). The composite material was reported to have high electrical conductivity, and showed an improved power factor.

The diameter and length of the Te nanowire fabricated using the aqueous solution method and the amount of PEDOT:PSS were controlled to produce the hybrid conditions necessary to achieve an optimal power factor (Fig. 6). According to this study, the electrical property of the hybrid was far greater than the electrical conductivities of the polymer and Te nanowire, and the hybrid had a ZT value of 0.1 at room temperature. Specifically, the electrical conductivity of the PEDOT:PSS was enhanced with no reduction in the Seebeck coefficient by doping with dimethylsulfoxide (DMSO). This was due to the conversion of the conducting polymer charge transport mechanism from the hopping transport to the carrier scattering mechanism. ${ }^{25)} \mathrm{Du}$ et al. used an intercalation/exfoliation method to remove p-type $\mathrm{Bi}_{0.5} \mathrm{Sb}_{1.5} \mathrm{Te}_{3}$, which has high thermoelectric properties at room temperature, in nanosheet form, to synthesize PEDOT: PSS hybrids with increased dispersion. In this case, the power factor increased to $32 \mu \mathrm{W} / \mathrm{mK}^{2}$, which was roughly 3 times that of the single phase PEDOT:PSS. This research result was significant in that it showed that the thermoelectric efficiency can be maximized through the organic-inorganic interfacial phenomena. ${ }^{26)} \mathrm{Ju}$ et al. used the ballmilling method to obtain SnSe in powder form and synthesized a SnSe nanosheet and PEDOT:PSS hybrid utilizing the Li-intercalation process. The interesting point in this study is that the electrical conductivity of the hybrid was not affected by the amount of SnSe nanosheets, and only the Seebeck coefficient was improved through the carrier filtering effect at the interface. Furthermore, an additional improvement in PEDOT:PSS electrical conductivity through DMSO de-doping increased the SnSe/PEDOT:PSS hybrid power factor up to $380 \mu \mathrm{W} / \mathrm{mK}^{2}{ }^{27)}$ In order to further improve 


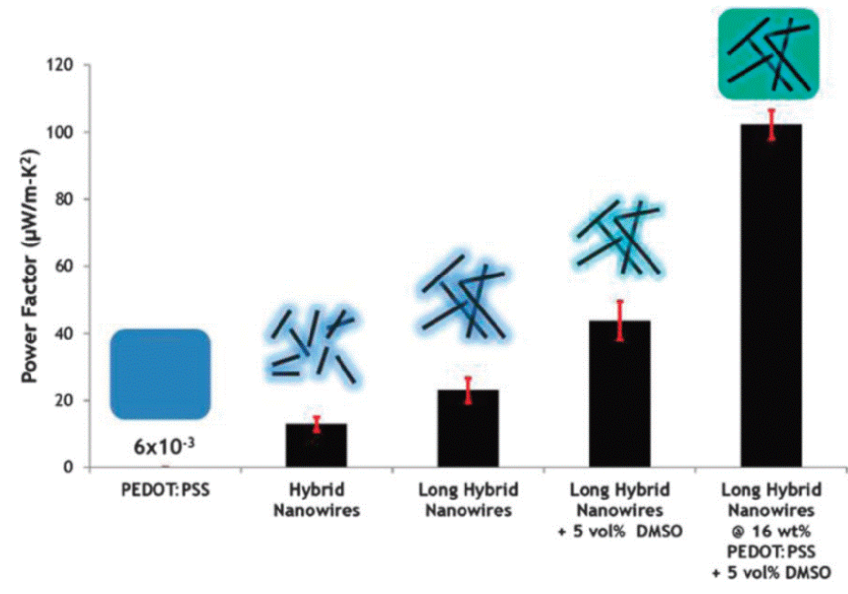

Fig. 6. Hybrid power factor improvement through Te nanowire/PEDOT:PSS synthesis.

such interfacial effects, Choi et al. synthesized a hybrid composed of a 3 phase graphene oxide (rGO)/PEDOT:PSS/Te nanowire. The thermoelectric property of the hybrid, which simultaneously had two types of energy barriers $(0.24 \mathrm{eV}$ Te/PEDOT:PSS, $0.31 \mathrm{eV}$ rGO/PEDOT:PSS), revealed a power factor of $143 \mu \mathrm{W} / \mathrm{mK}^{2}$ through the stronger carrier filtering effect at the interface, which was approximately 102 times that of the single phase. ${ }^{28)}$

\section{Conclusions}

In this study, the types and synthesis methods of organicinorganic thermoelectric hybrid materials were discussed along with the methods used to enhance their thermoelectric properties. In order to maximize the thermoelectric properties of organic-inorganic hybrids, nanoengineering can be utilized to control the nanostructure of the inorganic material, the organic material structure, and the doping level, to improve their thermoelectric properties. Also, the interface structure, density, and defects within the hybrid need to be considered to improve the thermoelectric properties, using organic-inorganic hybrid interfacial control. The interfacial control technology can lead to the development of thermoelectric modules with excellent high flexibility, high reliability, and high performance thermoelectric efficiency, based on hybrid organic/inorganic materials. Furthermore, the interfacial control technology is expected to become a key technology to maximize the efficiency of various electronic devices.

\section{Acknowledgements}

This research was funded by the Ministry of Knowledge Economy in 2015 and supported by the Korea Energy Technology Assessment Corporation (KETEP). (No. 20153030013200) Also, this research was supported by the Korea Institute of Materials Science (KIMS) under the Korea Institute of Machinery \& Materials (PNK5043, PNK 4670).

\section{REFERENCES}

1. G. J. Snyder and E. S. Toberer, "Complex Thermoelectric Materials," Nat. Mater., 7 [2] 105-14 (2008).

2. W. Xie, X. Tang, Y. Yan, Q. Zhang, and T. M. Tritt, "Unique Nanostructures and Enhanced Thermoelectric Performance of Melt-Spun BiSbTe Alloys," Appl. Phys. Lett., 94 [10] 102111 (2009).

3. S. I. Kim, K. Ahn, D.-H. Yeon, S. Hwang, H.-S. Kim, S. M. Lee, and K. H. Lee, "Enhancement of Seebeck Coefficient in $\mathrm{Bi}_{0.5} \mathrm{Sb}_{1.5} \mathrm{Te}_{3}$ with High-Density Tellurium Nanoinclusions," Appl. Phys. Express, 4 [9] 091801 (2011).

4. J. P. Heremans, V. Jovovic, E. S. Toberer, A. Saramat, K. Kurosaki, A. Charoenphakdee, S. Yamanaka, and G. J. Snyder "Enhancement of Thermoelectric Efficiency in $\mathrm{PbTe}$ by Distortion of the Electronic Density of States," Science, 321 [5888] 554-57 (2008).

5. M. Hong, T. C. Chasapis, Z.-G. Chen, L. Yang, M. G. Kanatzidis, G. J. Snyder, and J. Zou, "n-Type $\mathrm{Bi}_{2} \mathrm{Te}_{3-\mathrm{x}} \mathrm{Se}_{\mathrm{x}}$ Nanoplates with Enhanced Thermoelectric Efficiency Driven by Wide-Frequency Phonon Scatterings and Synergistic Carrier Scatterings," ACS Nano, 10 [4] 4719-27 (2016).

6. J. Carrete, N. Mingo, G. Tian, H. Ågren, A. Baev, and P. N. Prasad, "Thermoelectric Properties of Hybrid OrganicInorganic Superlattices,” J. Phys. Chem. C, 116 [20] 10881-86 (2012).

7. Y. H. Kim, C. Sachse, M. L. Machala, C. May, L. MüllerMeskamp, and K. Leo, "Highly Conductive PEDOT:PSS Electrode with Optimized Solvent and Thermal PostTreatment for ITO-Free Organic Solar Cells," Adv. Funct. Mater., 21 [6] 1076-81 (2011).

8. M. Martín-González, O. Caballero-Calero, and P. DíazChao, "Nanoengineering Thermoelectrics for 21st Century: Energy Harvesting and Other Trends in the Field," Renewable Sustainable Energy Rev., 24 288-305 (2013).

9. M. S. Dresselhaus, G. Chen, M. Y. Tang, R. G. Yang, H. Lee, D. Z. Wang, Z. F. Ren, J.-P. Fleurial, and P. Gogna, "New Directions for Low-Dimensional Thermoelectric Materials," Adv. Mater., 19 [8] 1043-53 (2007).

10. B. C. Sales, "Electron Crystals and Phonon Glasses: A New Path to Improved Thermoelectric Materials," MRS Bull., 23 [1] 15-21 (1998).

11. D.-K. Ko, Y. Kang, and C. B. Murray, "Enhanced Thermopower via Carrier Energy Filtering in Solution-Processable Pt-Sb $\mathrm{Te}_{3}$ Nanocomposites," Nano Lett., 11 [7] 284144 (2011).

12. D. L. Medlin and G. J. Snyder, "Interfaces in Bulk Thermoelectric Materials: A Review for Current Opinion in Colloid and Interface Science," Curr. Opin. Colloid Interface Sci., 14 [4] 226-35 (2009).

13. S. R. S. Kumar, N. Kurra, and H. N. Alshareef, "Enhanced High Temperature Thermoelectric Response of Sulphuric Acid Treated Conducting Polymer Thin Films," J. Mater. Chem. C, 4 [1] 215-21 (2016).

14. Q. Zhang, Y. Sun, W. Xu, and D. Zhu, "Organic Thermoelectric Materials: Emerging Green Energy Materials Converting Heat to Electricity Directly and Efficiently," Adv. Mater., 26 [40] 6829-51 (2014). 
15. Y. Sun, L. Qiu, L. Tang, H. Geng, H. Wang, F. Zhang, D. Huang, W. Xu, P. Yue, Y.-S. Guan, F. Jiao, Y. Sun, D. Tang, C.-A. Di, Y. Yi, and D. Zhu, "Flexible n-Type HighPerformance Thermoelectric Thin Films of Poly(nickelethylenetetrathiolate) Prepared by an Electrochemical Method," Adv. Mater., 28 [17] 3351-58 (2016).

16. Y. Du, S. Z. Shen, K. Cai, and P. S. Casey, "Research Progress on Polymer-Inorganic Thermoelectric Nanocomposite Materials," Prog. Polym. Sci., 37 [6] 820-41 (2012).

17. C. Gayner and K. K. Kar, "Recent Advances in Thermoelectric Materials," Prog. Mater. Sci., 83 330-82 (2016).

18. O. Bubnova, Z. U. Khan, A. Malti, S. Braun, M. Fahlman, M. Berggren, and X. Crispin, "Optimization of the Thermoelectric Figure of Merit in the Conducting Polymer Poly(3,4-ethylenedioxythiophene)," Nat. Mater., 10 [6] 429-33 (2011).

19. G. H. Kim, L. Shao, K. Zhang, and K. P. Pipe, "Engineered Doping of Organic Semiconductors for Enhanced Thermoelectric Efficiency," Nat. Mater., 12 [8] 719-23 (2013).

20. Q. Wang, Q. Yao, J. Chang, and L. Chen, "Enhanced Thermoelectric Properties of CNT/PANI Composite Nanofibers by Highly Orienting the Arrangement of Polymer Chains," J. Mater. Chem., 22 [34] 17612-18 (2012).

21. B. Cho, K. S. Park, J. Baek, H. S. Oh, Y. E. K. Lee, and M. M. Sung, "Single-Crystal Poly(3,4-ethylenedioxythiophene) Nanowires with Ultrahigh Conductivity," Nano Lett., 14 [6] 3321-27 (2014).

22. G. Lu, C. Li, J. Shen, Z. Chen, and G. Shi, "Preparation of Highly Conductive Gold-Poly(3,4-ethylenedioxythiophene)
Nanocables and Their Conversion to Poly(3,4-ethylenedioxythiophene) Nanotubes," J. Phys. Chem. C, 111 [16] 5926-31 (2007).

23. Y. Wang, K. Cai, and X. Yao, "Facile Fabrication and Thermoelectric Properties of PbTe-Modified Poly(3,4-ethylenedioxythiophene) Nanotubes," ACS Appl. Mater. Interfaces, 3 [4] 1163-66 (2011).

24. K. C. See, J. P. Feser, C. E. Chen, A. Majumdar, J. J. Urban, and R. A. Segalman, "Water-Processable PolymerNanocrystal Hybrids for Thermoelectrics," Nano Lett., 10 [11] 4664-67 (2010).

25. S. K. Yee, N. E. Coates, A. Majumdar, J. J. Urban, and R. A. Segalman, "Thermoelectric Power Factor Optimization in PEDOT:PSS Tellurium Nanowire Hybrid Composites," Phys. Chem. Chem. Phys., 15 [11] 4024-32 (2013).

26. Y. Du, K. F. Cai, S. Chen, P. Cizek, and T. Lin, "Facile Preparation and Thermoelectric Properties of $\mathrm{Bi}_{2} \mathrm{Te}_{3}$ Based Alloy Nanosheet/PEDOT:PSS Composite Films," ACS Appl. Mater. Interfaces, 6 [8] 5735-43 (2014).

27. H. Ju and J. Kim, "Fabrication of Conductive Polymer/ Inorganic Nanoparticles Composite Films: PEDOT:PSS with Exfoliated Tin Selenide Nanosheets for PolymerBased Thermoelectric Devices," Chem. Eng. J., 297 66-73 (2016).

28. J. Choi, J. Y. Lee, S. S. Lee, C. R. Park, and H. Kim, "High-Performance Thermoelectric Paper Based on Double Carrier-Filtering Processes at Nanowire Heterojunctions," Adv. Energy Mater., 6 [9] 1502181 (2016). 